\title{
Transcriptome profiling provides insights into dormancy release during cold storage of Lilium pumilum
}

\author{
Wang Wang ${ }^{\dagger}$, Xiaoxia Su${ }^{\dagger}$, Zhongping Tian, Yu Liu, Yunwei Zhou ${ }^{*}$ (1) and Miao He*
}

\begin{abstract}
Background: Bulbs of the ornamental flower Lilium pumilum enter a period of dormancy after flowering in spring, and require exposure to cold for a period of time in order to release dormancy. Previous studies focused mainly on anatomical, physiological and biochemical changes during dormancy release. There are no dormancy studies of the northern cold-hardy wild species of Lilium at the molecular level. This study observed bulb cell and starch granule ultrastructures during cold storage; and analysed the transcriptome using sequencing. The combination of morphological and transcriptomic methods provides valuable insights into dormancy release during cold storage of Lilium pumilum.

Results: Ultrastructural changes reflected dormancy release during cold storage of the bulbs. We compared gene expression levels among samples at 0 (S1 stage), 30 (S2 stage), 60 (S3 stage) and 90 (\$4 stage) d of cold storage, with $0 \mathrm{~d}$ as the control. The data showed that some regulatory pathways such as carbohydrate metabolism and plant hormone signal transduction were activated to break dormancy. Some differentially expressed genes (DEGs) related to antioxidant activity, epigenetic modification and transcription factors were induced to respond to low temperature conditions. These genes constituted a complex regulatory mechanism of dormancy release.

Conclusions: Cytological data related to dormancy regulation was obtained through histomorphological observation; transcriptome sequencing provided comprehensive sequences and digital gene expression tag profiling (DGE) data, and bulb cell ultrastructural changes were closely related to DEGs. The novel Lilium pumilum genetic information from this study provides a reference for the regulation of dormancy by genetic engineering using molecular biology tools.
\end{abstract}

Keywords: Low temperature - Lilium pumilum - transcriptome - dormancy release

\section{Background}

The dormancy of bulbs is influenced by many factors. The inhibition and induction model of hormonemediated regulation involves hormone-mediated inhibition of bud growth and anabolism, stimulation of shoot meristem growth, starch degradation and carbohydrate metabolism [1]. Abscisic acid (ABA) and gibberellin (GA) are important regulators of plant dormancy and germination, and they mitigate biotic and abiotic stress [2,3]. Ethylene (ETH) is a hormone that affects growth and development from germination to senescence [4]. Previous studies show that ETH breaks dormancy by antagonizing $\mathrm{ABA}$ and

\footnotetext{
*Correspondence: dlzhyw@126.com; hemiao_xu@126.com

${ }^{\dagger}$ Equal contributors

Northeast Forestry University, Harbin, China
}

promotes radicle processes [5]. Carbohydrate metabolism provides energy for starch hydrolysis and soluble sugar synthesis, which contributes to dormancy release. Energy metabolism also participates in dormancy regulation [6].

It has been found that reactive oxygen species (ROS) regulate both dormancy release and signal transduction [7, 8]. Many stress responsive genes (the transcription factors WRKY, CBF, MYB and $b Z I P$ ) are also involved in dormancy regulation [9-11]. Sequencing technology is a scientific means of exploring the molecular mechanisms of plant dormancy, and has been widely used in this field of research. Examples include transcriptome analyses of Japanese apricot buds using 454-pyrosequencing technology [12], Japanese pear [13], and Chinese white pear 'Suli' flower buds [14].

(c) The Author(s). 2018 Open Access This article is distributed under the terms of the Creative Commons Attribution 4.0 International License (http://creativecommons.org/licenses/by/4.0/), which permits unrestricted use, distribution, and reproduction in any medium, provided you give appropriate credit to the original author(s) and the source, provide a link to the Creative Commons license, and indicate if changes were made. The Creative Commons Public Domain Dedication waiver (http://creativecommons.org/publicdomain/zero/1.0/) applies to the data made available in this article, unless otherwise stated. 
Low temperature is an important environmental factor in bulb dormancy release and bud germination. During dormancy release, upstream and downstream factors regulate the transcription of many genes. Low temperatures influence the upstream regulation of GA biosynthesis, enhancing the biological activity of GA and thereby promoting dormancy release [15]. However, due to interspecific differences, dormancy types and treatment methods, there may be numerous differences in the effect of low temperature on dormancy. Dormancy release is related to the length of dormancy and the bulb storage method, and longer treatment times promote rapid germination [16].

Lilium pumilum originated from northern China and is an important, widely distributed, ornamental plant with rich nutritional value [17]. L. pumilum is highly resistant to Fusarium, leaf blight, drought, salinization and cold and is important for Lilium resistance breeding. In this study, we examined the ultrastructure and starch granules of bulb cells during cold storage. Cell morphology changes reflected the dormancy release process. Based on the cytological and physiological studies, a large number of dormancy-related genes were identified by transcriptome sequencing. This study enriches the genome information of L. pumilum, and provides valuable insights into dormancy release during cold storage of Lilium pumilum.

\section{Results}

\section{Ultrastructure of bulb cells during cold storage}

Figure 6 contains small text. Please provide replacement figure file. Otherwise, please confirm if we can retain the current presentation.The nuclear volumes did not change significantly after $60 \mathrm{~d}$ of cold storage, but the nucleoli became compacted and clear (Fig. 1a, b, c). After $90 \mathrm{~d}$, the nuclei were larger, and the nucleoli were loose (Fig. 1d). At harvest (S1), bulb cells had abundant mitochondria with a small number of visible tubular bubble crests (Fig. 2a), reduced Golgi, and fewer secretory vesicles (Fig. 3a). After $30 \mathrm{~d}$ of cold storage, mitochondria became elongated (Fig. 2b), and Golgi bodies consisted of 3-5 layers of stacked flat vesicles (Fig. 3b). After $60 \mathrm{~d}$ of cold storage, many spherical mitochondria were observed (Fig. 2c), and Golgi vesicles of bulb cells were stacked up to 6-7 layers, and large vacuoles appeared near the nuclei (Fig. 3c). There were no significant changes in plastids from 0 to $60 \mathrm{~d}$. After $90 \mathrm{~d}$ of cold storage, many spherical mitochondria were still visible (Fig. 2d); Golgi vesicles were stacked up to

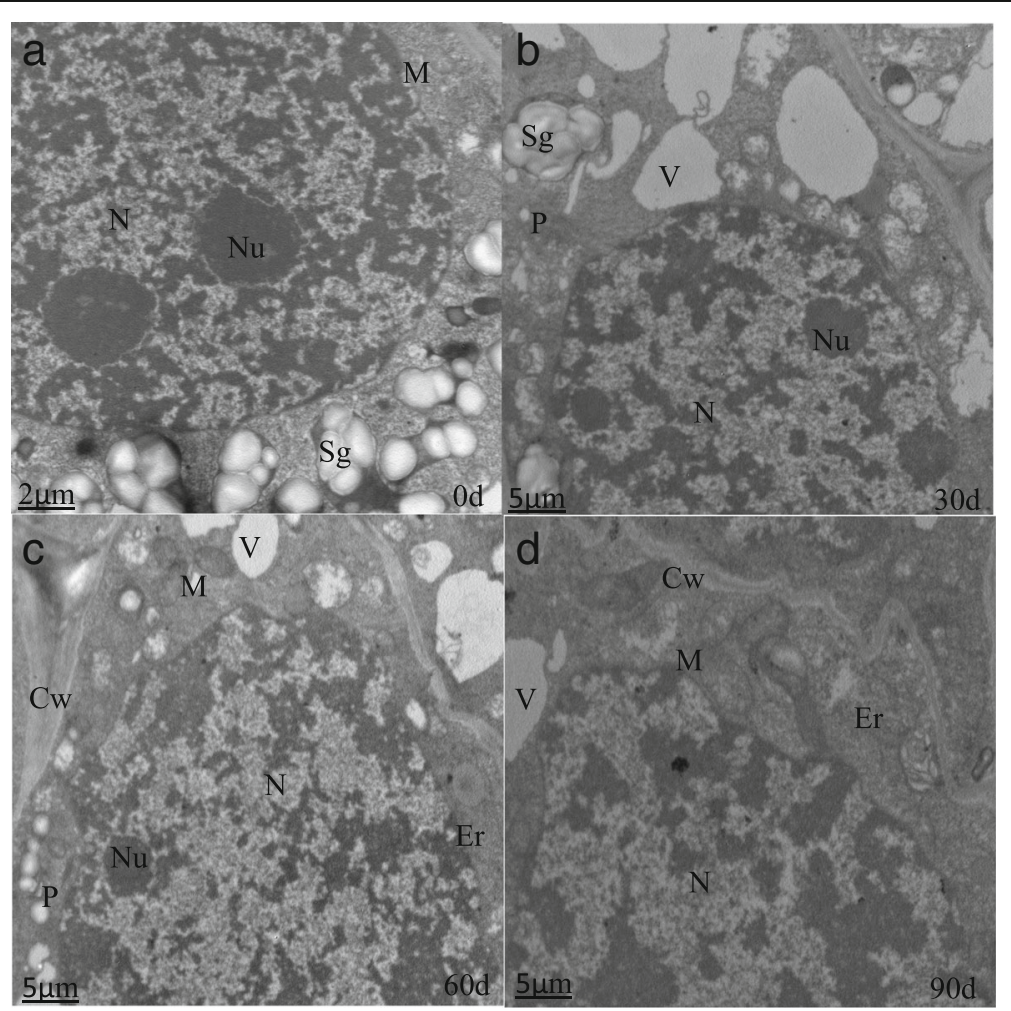

Fig. 1 The nuclear ultrastructure in the apical bud of Lilium pumilum during cold storage. $\mathbf{a}, \mathbf{b}, \mathbf{c}$ and $\mathbf{d}$ represent the nuclear ultrastructure of the apical bud at $0 \mathrm{~d}, 30 \mathrm{~d}, 60 \mathrm{~d}$ and $90 \mathrm{~d}$ of cold storage, respectively. Cw, Cell wall; Er, Endoplasmic reticulum; M, Mitochondrion; N, Cell nucleus; Nu, Nuclei; P, Plastid; Sg, Starch granule; V, Vacuole 


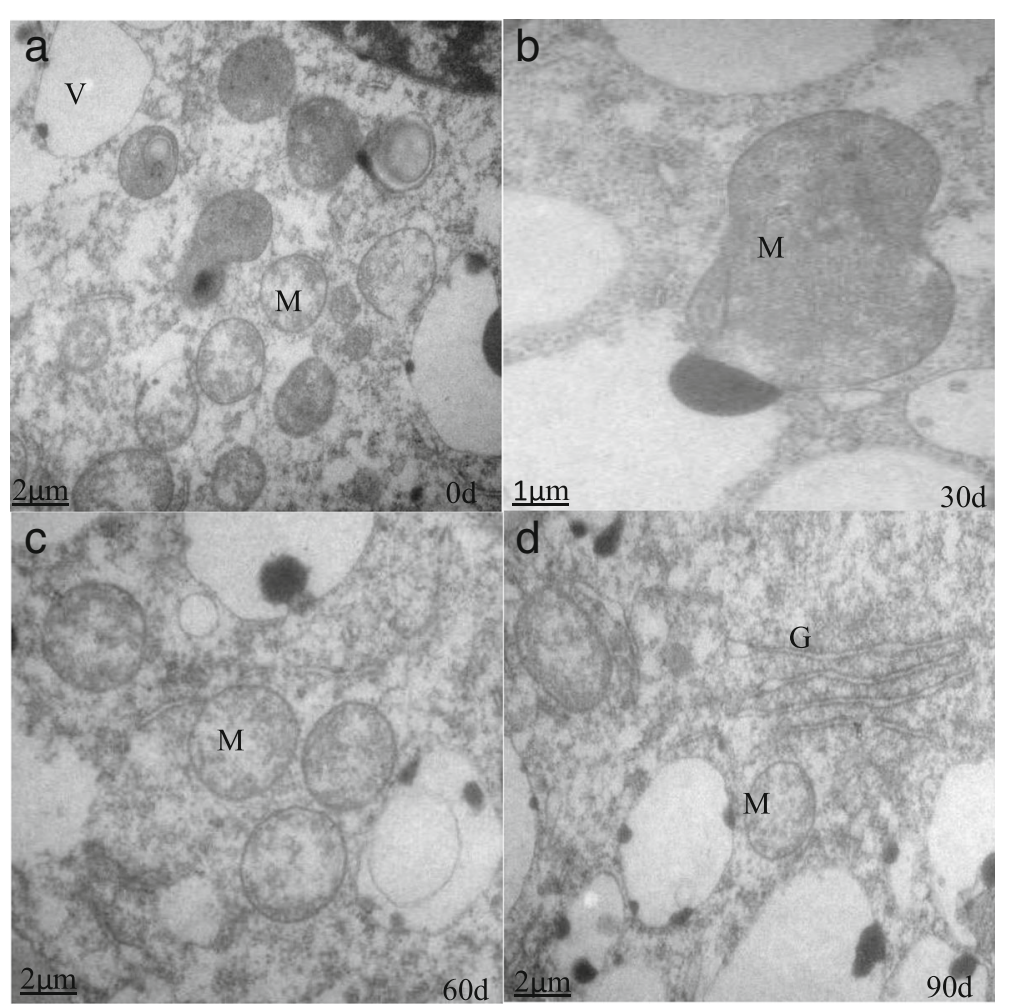

Fig. 2 The mitochondria ultrastructure in the apical bud of Lilium pumilum during cold storage. $\mathbf{a}, \mathbf{b}, \mathbf{c}$ and $\mathbf{d}$ represent mitochondria ultrastructure in the apical bud at $0 \mathrm{~d}, 30 \mathrm{~d}, 60 \mathrm{~d}$ and $90 \mathrm{~d}$ of cold storage, respectively. G, Golgi body; M, Mitochondrion; V, Vacuole

8-10 layers (Fig. 3d); more small and large vacuoles and irregular shaped plastids appeared in the cells; and there was a marked increase in numbers of long linear endoplasmic reticula (Fig. 1d).

Parenchyma cells were completely filled with starch granules upon initial harvest (Fig. 4), which significantly decreased after $90 \mathrm{~d}$ of cold storage.

The numbers and types of organelles increased to regulate the intracellular environment and perform a variety of biological functions. Mitochondria are the main sites for cellular aerobic respiration, and they provide energy for dormancy release and regulate cell growth and the cycle during cold storage. The Golgi and endoplasmic reticulum constitute the important endometrial system. The degradation of starch granules provides a source of energy for various biological processes. The structure and function of the organelles are interdependent, and based on the ultrastructural changes, we infer that dormancy release occurs during cold storage.

\section{Transcriptome sequencing of bulbs during cold storage Sequencing data}

A total of $62.04 \mathrm{~Gb}$ of high quality data (four developmental stages and three biological replicates, the raw reads files were deposited in the NCBI Sequences Read Archive under SRP108930) was obtained after filtration, and after de novo assembly, the data were assembled with trinity to yield 189,271 transcripts and 80,193 unigenes. The N50 of the transcripts and the unigenes were 1540 and 1228, respectively, indicating high assembly quality. The unigene length distribution is shown in Additional file 1 . Unigene sequences were compared and annotated with $\mathrm{nr}$ (ftp://ftp.ncbi.nih.gov/blast/db/), Swiss Prot (https://www.uniprot.org/), GO (http://www.geneontology.org/), COG (http://www.ncbi.nlm.nih.gov/COG/), KOG (ftp://ftp.ncbi.nih.gov/pub/COG/KOG/kyva), Pfam (http://pfam.xfam.org/) and KEGG (http://www.genome.jp/kegg/) databases (Additional file 2).

\section{Analysis of DEGs}

DEGs were significantly enriched during cold storage (Table 1). There were more DEGs in S1 vs S4 relative to S1 vs $\mathrm{S} 2$ and $\mathrm{S} 1$ vs $\mathrm{S} 3$, suggesting a greater complexity for the regulation of dormancy in S4. The hierarchical cluster and distribution of DEGs are shown in Fig. 5.

\section{GO enrichment analysis of DEGs}

There were 65,892 unigenes that were annotated in the GO database. These unigenes were divided into three subcategories: biological process, accounting for 45.6\%; cellular component, accounting for 22.4\%; and molecular function, 


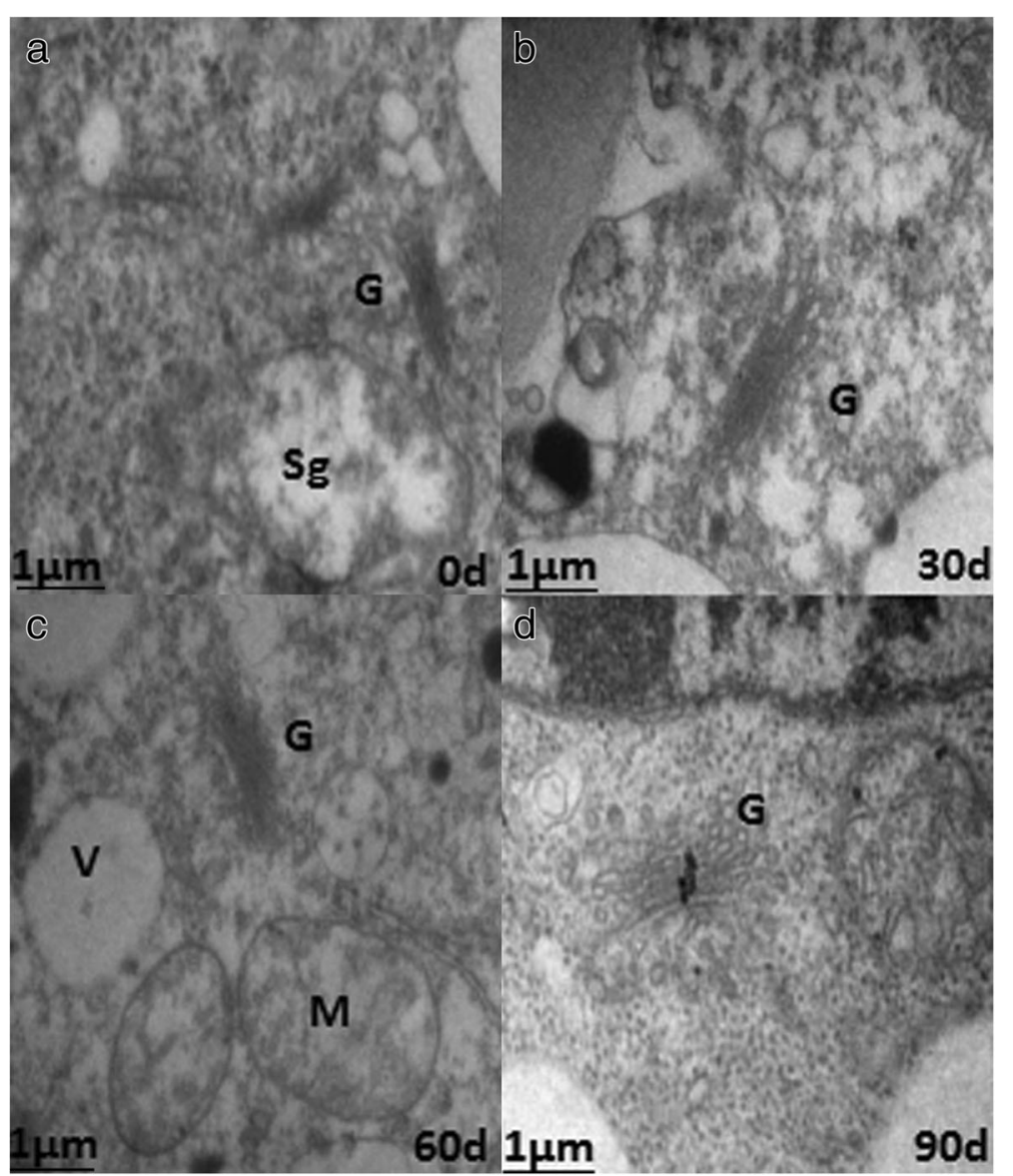

Fig. 3 Ultrastructure of Golgi bodies in the apical bud of Lilium pumilum during cold storage. $\mathbf{a}, \mathbf{b}, \mathbf{c}$ and $\mathbf{d}$ represent ultrastructure of Golgi bodies in the apical bud at $0 \mathrm{~d}, 30 \mathrm{~d}, 60 \mathrm{~d}$ and $90 \mathrm{~d}$ of cold storage, respectively. G, Golgi body

accounting for $32 \%$. There were 2,337 and 764 and 3,585 DEG unigenes in S1 vs S2, S1 vs S3 and S1 vs S4, respectively (Fig. 6, Additional file 3). Representative subclasses such as metabolic processes and catalytic activity, were significantly enriched, which implies that activation of energy metabolism pathways and related enzymes are required for dormancy release. Enrichment also occurred for genes associated with cellular processes, such as cell, cell part and cellular process. Many cells divide and grow after dormancy release, and the number and volume of cells increase during vegetative growth.

\section{KEGG enrichment analysis of DEGs}

In total, 5596 genes including 119 pathways were annotated in the KEGG database, of which ribosome (ko03010), ribosome biogenesis in eukaryotes (ko03008), RNA degradation (ko03018), spliceosome (ko03040) and RNA transport (ko03013) annotated the most genes.The DEG unigenes in S1 vs S2, S1 vs S3 and S1 vs S4 were involved in 64, 43 and 80 pathways, respectively (Fig. 7, Additional file 4). We found some representative secondary metabolic pathways, such as phenylpropanoid biosynthesis and flavonoid biosynthesis. Genes encoding enzymes in biosynthetic pathways, such as flavonol synthase, chalcone synthase and chalcone isomerase were down-regulated during cold storage. Secondary metabolites are defensive compounds and may consume plant nutrients during their accumulation. The down-regulation of secondary metabolite-related genes may be a selfprotection strategy against various abiotic stresses. Starch and sucrose metabolism as well as plant hormone signal transduction were also enriched. The former regulates plant energy metabolism, while the latter regulates endogenous hormone synthesis. These metabolic pathways interact to constitute a complex dormancy regulation network.

\section{DEGs involved in starch and sucrose metabolism pathway during dormancy release}

Cold acclimation is a complex process that involves a wide range of plant metabolic groups. Carbohydrate metabolism is an important component of the reprogrammed metabolic group especially under low temperature conditions, and the conversion of stored starch into soluble carbohydrates during cold acclimation has been widely 


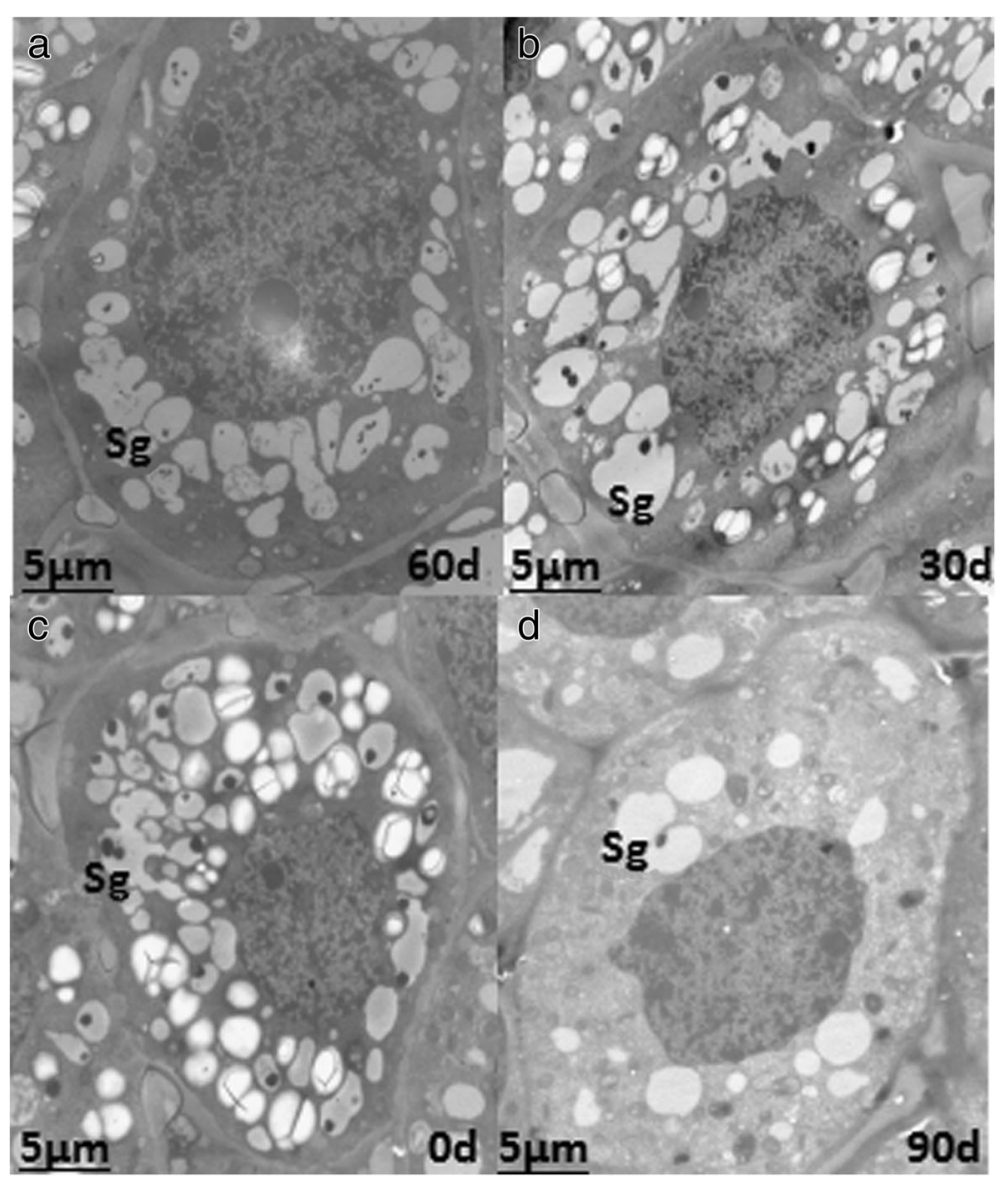

Fig. 4 Starch granule changes in apical cells. a, b, c and d represent starch granules at 0 d, 30 d, 60 d and 90 d of cold storage, respectively.Sg, Starch granule

reported in various plant species [18]. There were 829 unigenes involved in carbohydrate metabolism pathways, including starch and sucrose metabolism, glycolysis/ gluconeogenesis, amino sugar and nucleotide sugar metabolism, and pyruvate metabolism (Fig. 8), of which, starch and sucrose metabolism accounted for the largest proportion (134/829). We focus on the differential expression of $\alpha$-amylase (AMY) and $\beta$-amylase (BMY) related genes, only c111346.graph_c1(AMY) and c114414.graph_c0 (BMY) were expressed in S1 vs S2; most of the DEGs were enriched in S1 vs S4 and many were up-regulated (Table 2, Additional file 5). Meanwhile, we found that the DEGs involved in sucrose synthesis were also up-regulated, including c93866.graph_c0 and c94446.graph_c0. During the dormancy, the carbohydrates

Table 1 The number of DEGs

\begin{tabular}{llll}
\hline DEGs Set & All DEGs & up-regulated & down-regulated \\
\hline S1 vs S2 & 980 & 477 & 503 \\
S1 vs S3 & 294 & 90 & 204 \\
S1 vs S4 & 1683 & 726 & 957 \\
\hline
\end{tabular}

were mainly present in the form of starch, and at the later stage of the low temperature storage, starch hydrolysis accompanied by accumulation of sucrose released the metabolic energy for bulb dormancy release [19].

\section{DEGs involved in energy metabolism pathway during dormancy release}

There were 121, 61 and 38 unigenes involved in glycolysis/gluconeogenesis, the citrate cycle (TCA cycle) and the pentose phosphate pathway (PPP), respectively, which are the three main pathways for plant energy metabolism. The DEG encoding pyruvate dehydrogenase E1 (c110227.graph_c0), which is responsible for entry of pyruvate into the TCA cycle, was only expressed in S1 vs S2 (Table 2, Additional file 5); perhaps it is mainly involved in the transition from S1 to S2 stage. The other enzymes involved in subsequent steps, including 2oxoglutarate dehydrogenase (c110805.graph_c0) and isocitrate dehydrogenase (c89446.graph_c), were upregulated in S1 vs S4, and the TCA cycle may be activated in the later stage of cold storage. The key enzymes encode the genes ATP-dependent 6-phosphofructokinase 3 
a

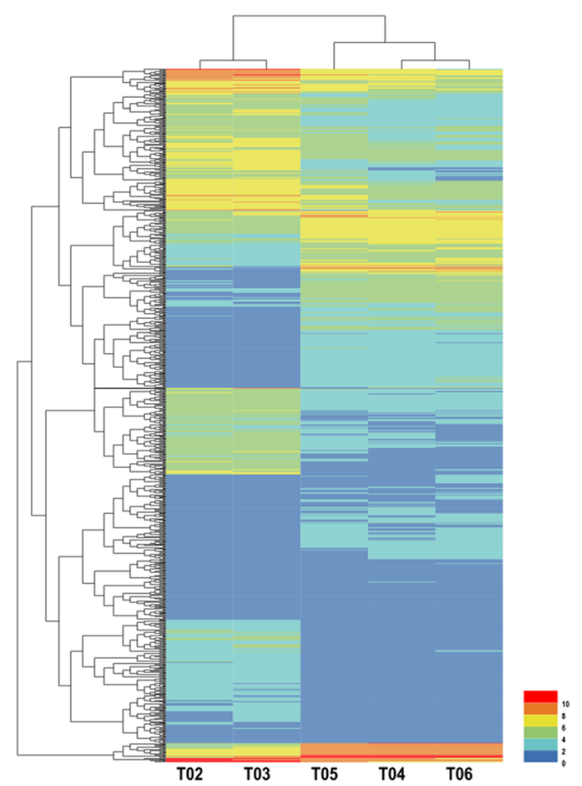

C

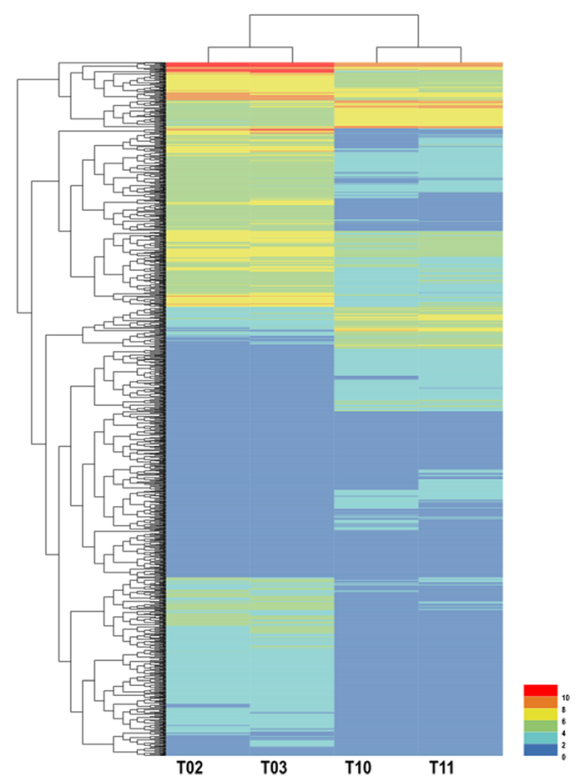

b

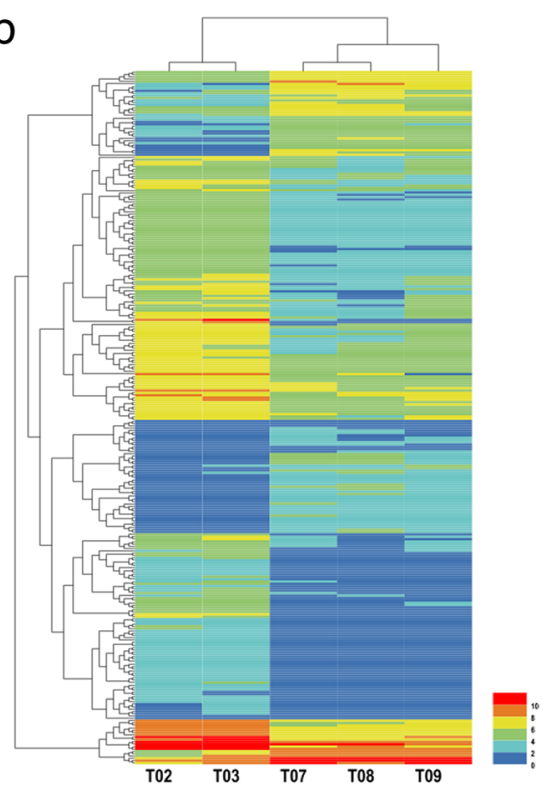

d

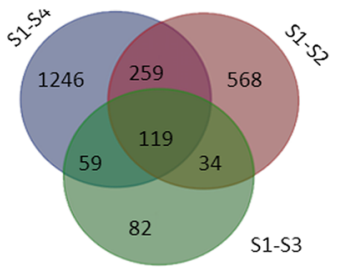

Fig. 5 Hierarchical cluster and distribution of DEGs. a, b and c represent the cluster heat map in S1 vs S2, S1 vs S3 and S1 vs S4, respectively. d Venn diagram of DEGs

(c97761.graph_c0), ATP-dependent 6-phosphofructokinase 2 (c101812.graph_c0), 6-phosphogluconolactonase 4 (c102 778.graph_c0) and ribose-5-phosphate isomerase (c953 01.graph_c0), which are involved in PPP, and the up - regulation of the DEGs indicated that the PPP is induced by cold treatment. In glycolysis/gluconeogenesis, some of the DEGs were up-regulated, including ATP-dependent 6phosphofructokinase 3 (c97761.graph_c0) and 2,3-bisphosphoglycerate-independent phosphoglycerate mutase (c7600 5 .graph_c0). However, the down-regulated DEGs accounted for a greater proportion.

\section{DEGs involved in hormone signal transduction pathway} during dormancy release

A variety of plant hormones interact to constitute a complex dormancy regulation network. ABA is necessary to induce plant dormancy and inhibit germination, while GA promotes germination [20]. The carotenoid metabolism pathway was associated with ABA metabolism, and 9-cis-epoxycarotenoid dioxygenase (NCED) and carotenoid cleavage dioxygenase 4 (CCD) regulated ABA synthesis. Many of the DEGs were mainly enriched in $\mathrm{S} 1$ vs $\mathrm{S} 4$ and all of them were down-regulated (Table 2, 

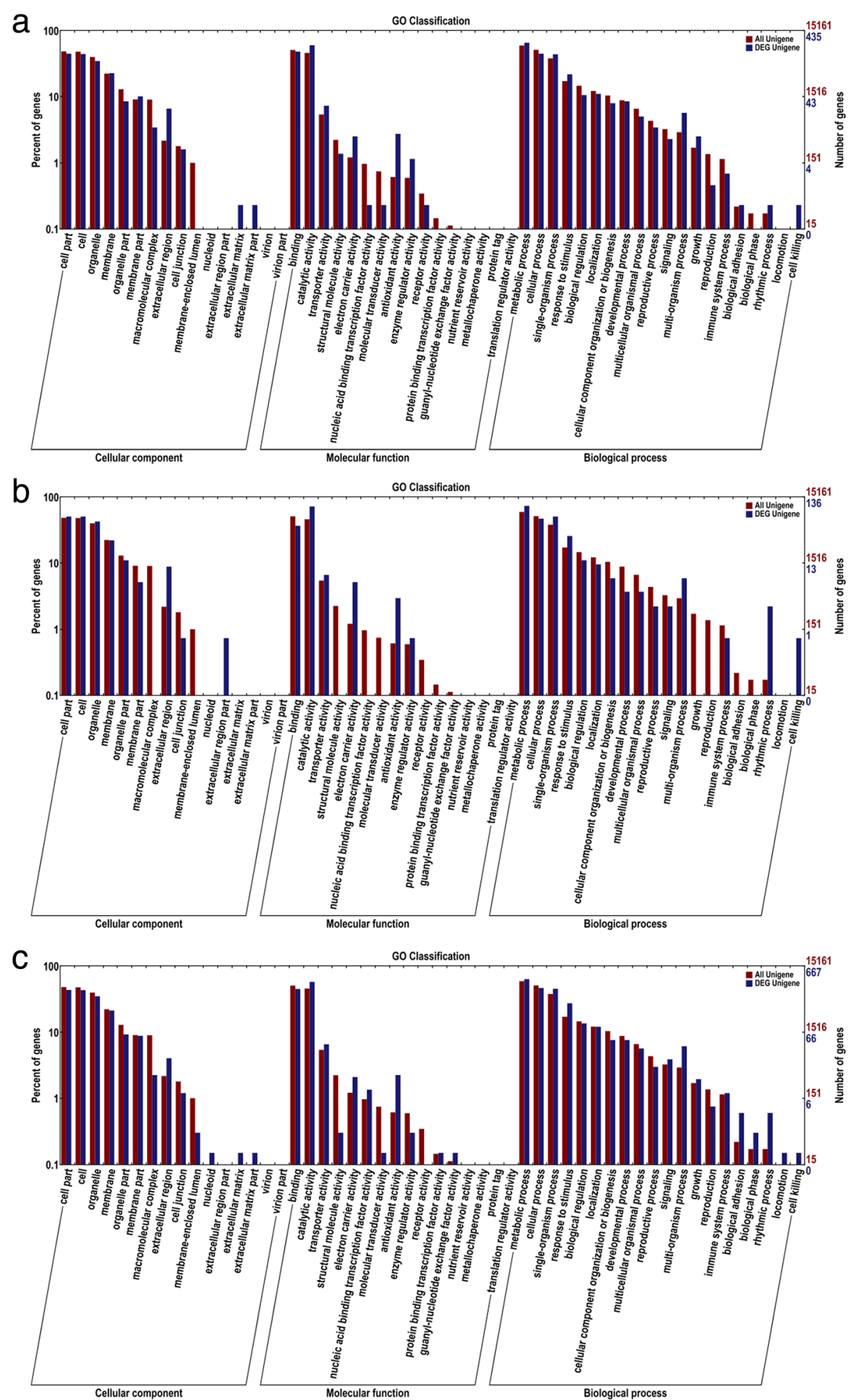

Fig. 6 GO enrichment diagrams. a, b and c represent the secondary GO term annotation for DEGs in S1 vs S2, S1 vs S3 and S1 vs S4, respectively

Additional file 5). Correspondingly, the DEGs encoding abscisic acid 8\&apos;-hydroxylase 2 (CYP707A), which are involved in ABA degradation, were all up-regulated in
S1 vs S3. ABA concentrations may be reduced during low temperature storage. The diterpenoid biosynthesis pathway was associated with GA metabolism. Gibberellin 2-beta- 

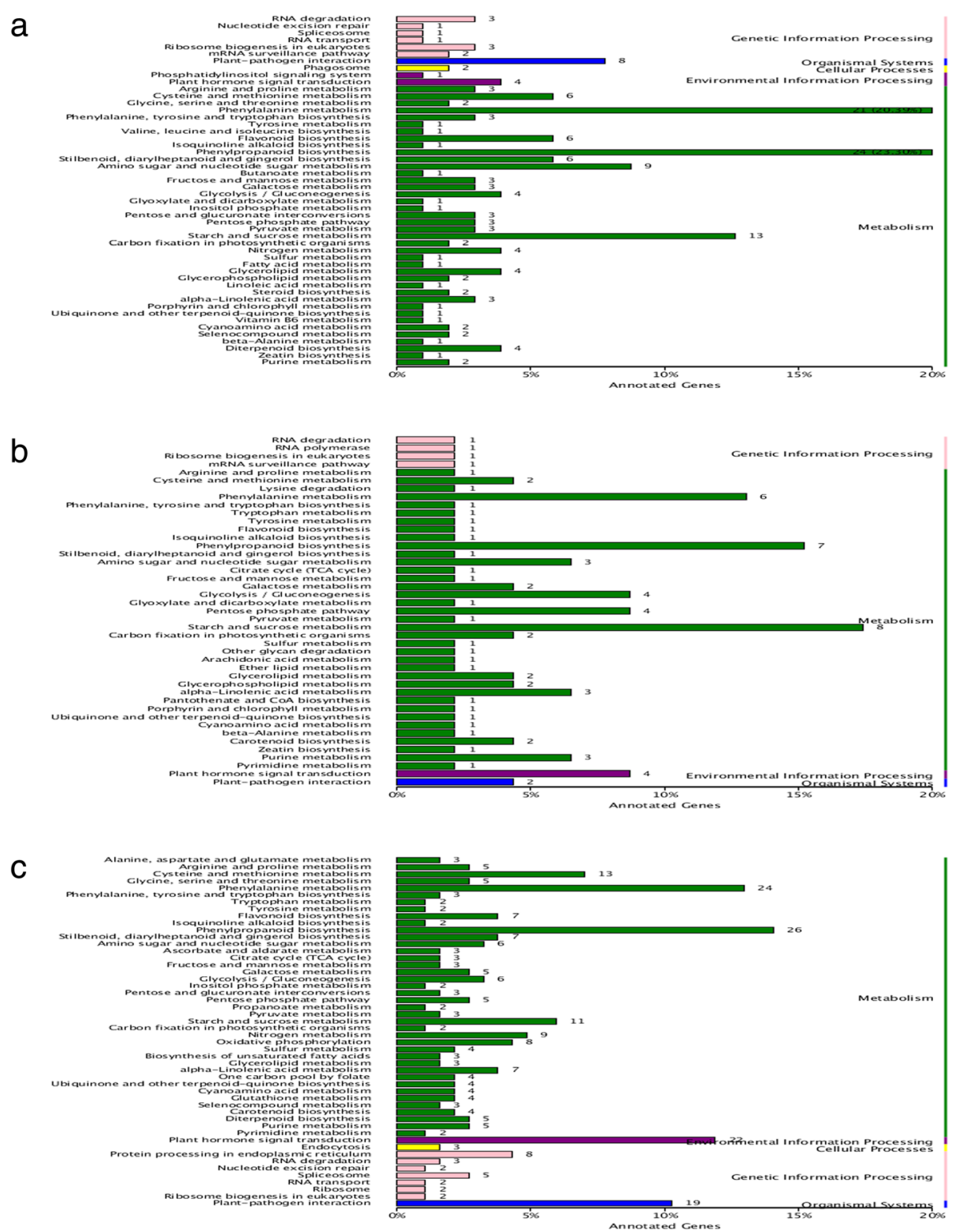

Fig. 7 KEGG enrichment diagrams. a, b and c represent the KEGG categories for genes in S1 vs S2, S1 vs S3 and S1 vs S4, respectively

dioxygenase(GA2ox1) and gibberellin 3-beta-dioxygenase (GA3ox4) were related to five down-regulated DEGs that inactivate GA; however, none of the DEGs encoding gibberellin 20 oxidase (GA20ox) that participated in GA synthesis were found. We further analysed the data and found that there were six genes encoding GA20ox (not listed); these genes regulate GA synthesis, but the roles did not show a difference in different stages of cryogenic storage. The ACC oxidase transcript (c97695.graph_c0), which oxidizes ACC to ETH, was only expressed in S1 vs S4 and up-regulated, thereby increasing the ETH levels. Alpha-linolenic acid metabolism and phenylalanine biosynthesis are related to the jasmonic acid (JA) and salicylic acid (SA) biosynthesis pathways. However, the DEGs encoding linoleate 13Slipoxygenase and phenylalanine ammonia-lyase, which are involved in JA and SA synthesis, respectively, were enriched in S1 vs S2 and S1 vs S4, all of them were down- regulated. The finding was consistent with the study of Glenn et al. [21].

\section{DEGs involved in antioxidant reactions during dormancy release}

The equilibrium between oxidation and the antioxidant system is shifted by plant cell respiration and energy metabolism, which promotes the accumulation of ROS and leads to oxidative stress. When the concentration of ROS is sufficient, bulb dormancy can be relieved [22]. 

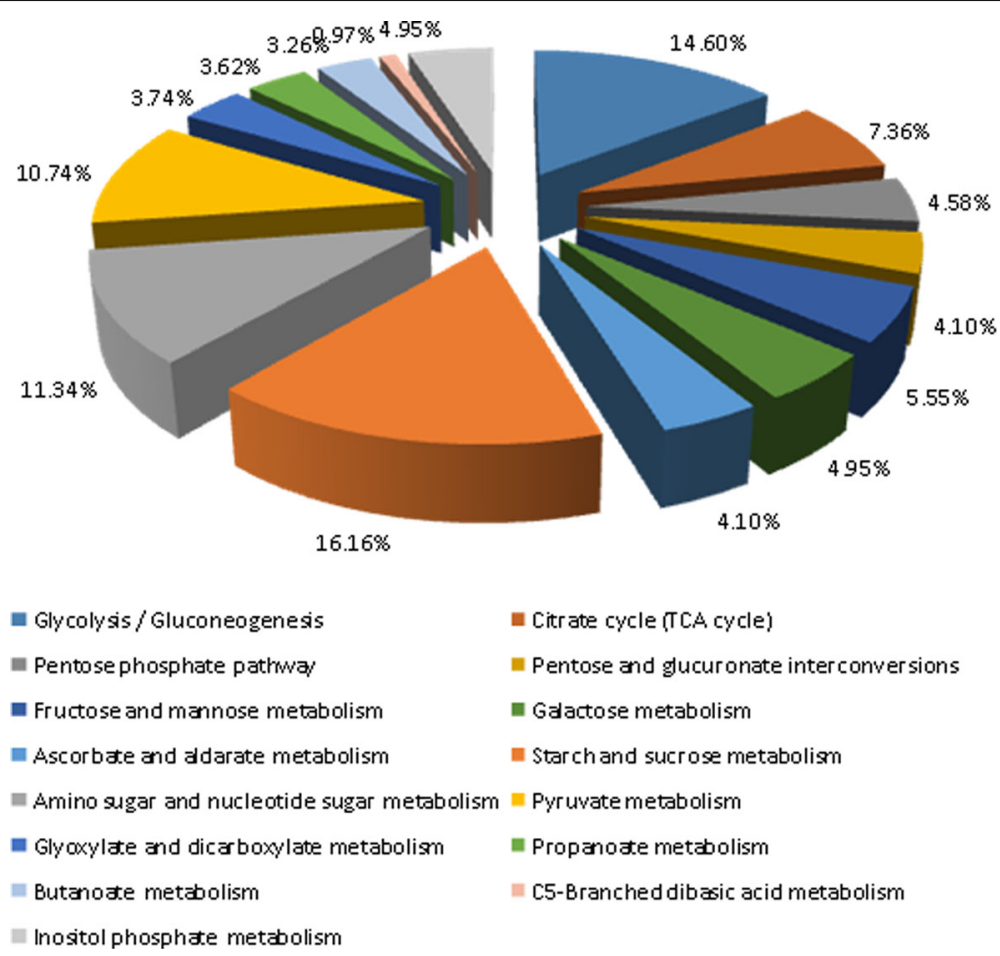

Fig. 8 The unigenes distribution profiles involved in carbohydrate metabolism pathways

Several key enzymes were found to be involved in the antioxidant reaction, such as catalase (CAT), alcohol dehydrogenase $(\mathrm{ADH})$, stilbene synthase (StSy) and glutathione S-transferase (GST) (Table 3, Additional file 6). Our data showed that the transcript of CAT (c104193.graph_c1) was reduced in S1 vs S4. The downregulation of CAT could increase the hydrogen peroxide content to trigger dormancy release [23]. The DEGs encoding $\mathrm{ADH}$ were only enriched in S1 vs S4, of which, c106934.graph_c0 was up-regulated. The induction of the ADH gene could reduce the oxidative phosphorylation pathway induced by cold treatment and artificial stimuli [24]. Antioxidative machinery such as StSy and GST were significantly enriched in S1 vs S4; many of these genes were induced, which indicated that the ascorbate-glutathione cycle was probably induced to

Table 2 The DEG unigenes involved in dormancy release pathways

\begin{tabular}{|c|c|c|c|c|c|c|}
\hline \multirow[t]{2}{*}{ DEG Unigene } & \multicolumn{2}{|c|}{ S1 vs S2 } & \multicolumn{2}{|c|}{ S1 vs S3 } & \multicolumn{2}{|c|}{ S1 vs S4 } \\
\hline & up & down & up & down & up & down \\
\hline \multicolumn{7}{|l|}{ Starch and sucrose metabolism } \\
\hline Starch degradation & 0 & $100 \%$ & $67 \%$ & $33 \%$ & $57 \%$ & $43 \%$ \\
\hline Sucrose synthesis & 0 & 0 & $100 \%$ & 0 & $100 \%$ & 0 \\
\hline \multicolumn{7}{|l|}{ Energy Metabolism } \\
\hline Glycolysis /Gluconeogenesis & 0 & $100 \%$ & $33 \%$ & $67 \%$ & $33 \%$ & $67 \%$ \\
\hline PPP & $33 \%$ & $67 \%$ & $33 \%$ & $67 \%$ & $60 \%$ & $40 \%$ \\
\hline TCA cycle & 0 & $100 \%$ & $100 \%$ & 0 & $67 \%$ & $33 \%$ \\
\hline \multicolumn{7}{|l|}{ Plant hormone signal transduction } \\
\hline$A B A$ & 0 & $100 \%$ & $67 \%$ & $33 \%$ & 0 & $100 \%$ \\
\hline GA & 0 & $100 \%$ & 0 & 0 & 0 & $100 \%$ \\
\hline ETH & 0 & 0 & 0 & 0 & $100 \%$ & 0 \\
\hline$J A$ & 0 & $100 \%$ & 0 & 0 & 0 & $100 \%$ \\
\hline SA & 0 & $100 \%$ & 0 & 0 & 0 & $100 \%$ \\
\hline
\end{tabular}


Table 3 The DEG unigenes involved in cellular activity

\begin{tabular}{|c|c|c|c|c|c|c|}
\hline \multirow[t]{2}{*}{ DEG Unigene } & \multicolumn{2}{|c|}{ S1 vs S2 } & \multicolumn{2}{|c|}{ S1 vs S3 } & \multicolumn{2}{|c|}{ S1 vs S4 } \\
\hline & up & down & up & down & up & down \\
\hline Antioxidant reaction & $100 \%$ & 0 & $100 \%$ & 0 & $42 \%$ & $58 \%$ \\
\hline DNA methylation & 0 & 0 & 0 & 0 & 0 & $100 \%$ \\
\hline Cell division and cell growth & $67 \%$ & $33 \%$ & 0 & 0 & $86 \%$ & $14 \%$ \\
\hline
\end{tabular}

cope with the increased oxidative stress in the bud at the later stage of low temperature storage [6].

\section{DEGs involved in DNA methylation during dormancy release}

DNA methylation increased throughout the genome in an ABA-dependent manner [25]. However, DNA demethylation occurred prior to transcriptional activation of genes involved in cell division and meristematic growth [26]. We focused on the biological process related to DNA methylation (GO:0006306) and DNA demethylation (GO:0080111), in total, 68 and 1 genes were annotated, respectively. Of which, two DEGs related to DNA (cytosine-5)-methyltransferase were downregulated in S1 vs S4 (Table 3, Additional file 6). Low temperature affects epigenetic modification through DNA methylation [27]. Transcriptional silencing triggered by DNA methylation may lead to growth arrest during dormancy; the reduction of DNA methylation may respond to dormancy release at the later stage of low temperature storage. However, the genes involved in DNA demethylation were not differentially expressed.

\section{DEGs involved in cell division and growth during dormancy release}

Plant growth and development remain stagnant during dormancy, while cell division is accelerated to meet the needs of vegetative growth after dormancy release [28]. There were 53 and 39 genes that participated in cell division (GO:0051301) and cell growth (GO:0016049) biological processes. All of the DEGs were enriched in S1 vs S4 and many of them were up-regulated (Table 3, Additional file 6). Meanwhile, cyclin-A and cyclin-D, were closely related to the mitotic activity of the shoot meristem [29]. In our study, c77281.graph_c0 encoded cyclin-A and c104987.graph_c0 encoded cyclin-D, both of them were up-regulated to stimulate mitotic activity in response to the dormancy release. Cell mitosis is also the cause of the increase in the number and volume of cells to meet the need for vegetative growth after bulb dormancy release.

\section{QRT-PCR validation of DEGs}

To verify the results of the transcriptome data, 14 DEGs were randomly selected for qRT-PCR analysis (Fig. 9), the primer information was shown in Additional file 7.
Most of the transcriptome data agreed with the qRTPCR results; the few inconsistencies may have been due to the differences in the sensitivity, specificity, and the algorithms for qRT-PCR and sequencing technology.

\section{Transcription factors responding to abiotic stimulus}

The cold acclimation process is accompanied by a variety of abiotic stimuli. Cold temperatures trigger the expression of the $C B F$ family of transcription factors, which in turn activate many downstream genes that confer freezing tolerance to plants [30]. However, $C B F$ was not enriched, and only one was found during cold storage (Fig. 10). Bai et al. [13] observed the same results with a transcriptome analysis of Japanese pear flower buds transitioning through endodormancy. The result is beyond our expectation. Although the important role of low temperature in dormancy release is widely recognized, how the frozen signal is sensed and transduced to the downstream factors has been in the discussion. In Arabidopsis, MYB participates in cold stress, regulates ABA-responsive genes and plays an important role in the upstream step of cold stress signalling transduction [31]. The WRKY gene family plays important roles in abiotic stress tolerance and ABA- signalling [32]. WRKY is known to promote the expression of anti-freezing protein (AFP) in response to abiotic stress [33]. MYB and WRKY were up-regulated in the early cryopreservation (Fig. 10), and they act as potential upstream regulators in freezing or may be involved in tolerance to multiple stresses. In addition, $b Z I P$ and DREB also regulate the expression of many stress-inducible genes, mostly in an ABAindependent manner, and play key roles in improving plant abiotic stress tolerance [34, 35]. Our data showed that $b Z I P$ and $D R E B$ were significantly enriched (Fig. 10).

\section{Discussion}

Low temperature is an important environmental signal for dormancy release. We identified a large number of DEGs comprising a complex network of dormancy regulation. The study provides insight into the mechanism of L. pumilum dormancy and has added greatly to the molecular data for bulbous plants.

\section{Changes in bulb cell ultrastructure during low temperature storage}

The plant cell ultrastructural changes are closely related to the process of dormancy release. A previous study found that in the seasonal dormancy buds of poplar, the cell wall thickens, the storage of protein in the vacuole increases, and contraction and blocking of the intercellular connection leads to interruption of the transport of the comonomer, limiting the exchange and signal transduction between adjacent cells, which in turn leads to growth arrest and dormancy [36]. And 


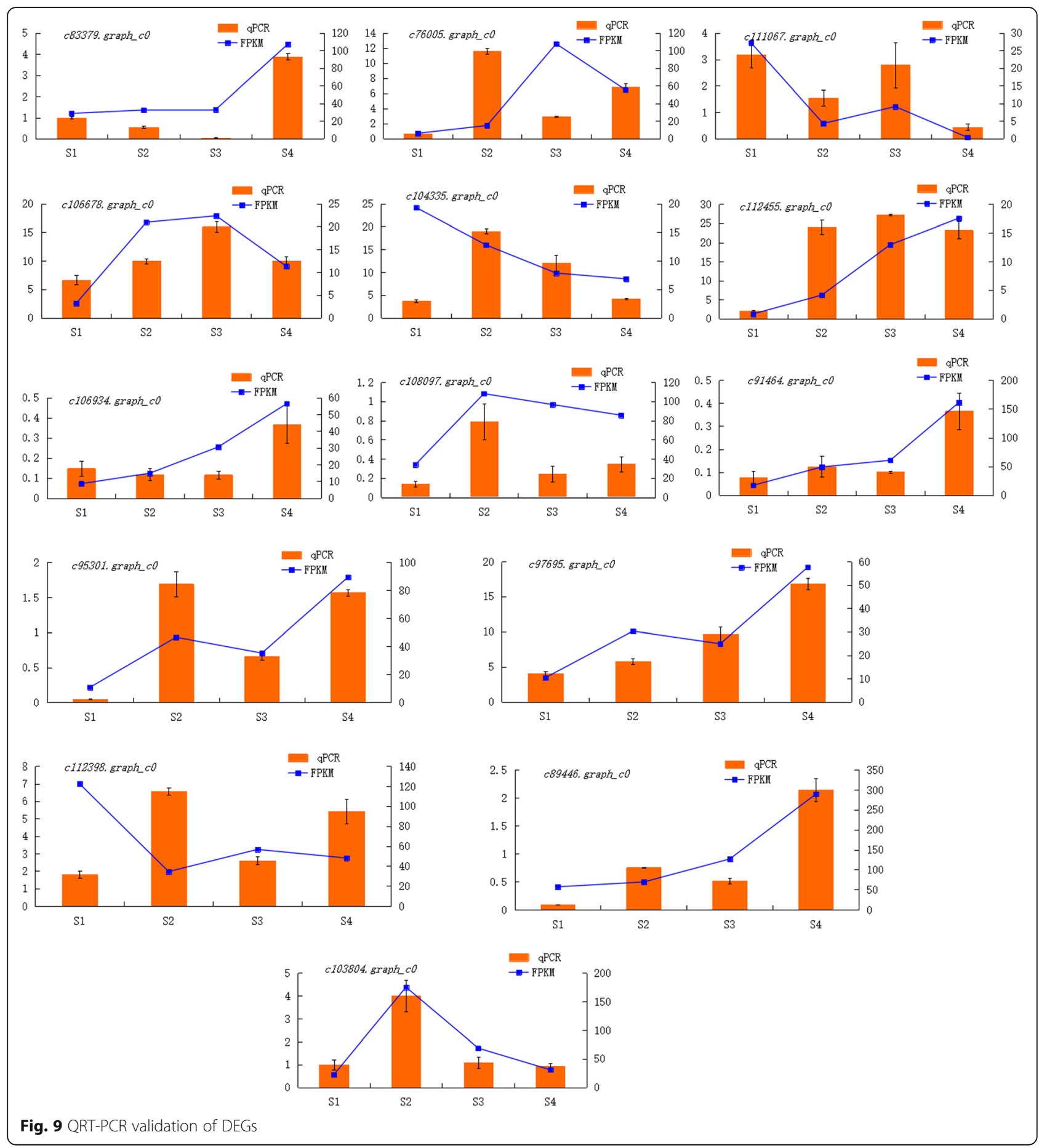

increase in the number of organelles, such as mitochondria, plastids, Golgi bodies, vacuoles and micro bodies, in the apical meristem cells occurs when dormancy is broken [37]. Cold treatment relieves the inhibition of specific transcription and translation sequences, leading to structural changes in stem cells [38]. With the prolongation of low temperature storage, the nuclei volume increased. The nucleus plays an important role in metabolism, growth and differentiation of cells and is the main site of genetic material [39]. The type and number of organelles were constantly enriched, including endoplasmic reticulum, mitochondrion, plastids, vacuoles and Golgi bodies. Mitochondria are the main place for aerobic respiration, and the energy released satisfies that needed for dormancy release [40]. Golgi bodies constitute the 


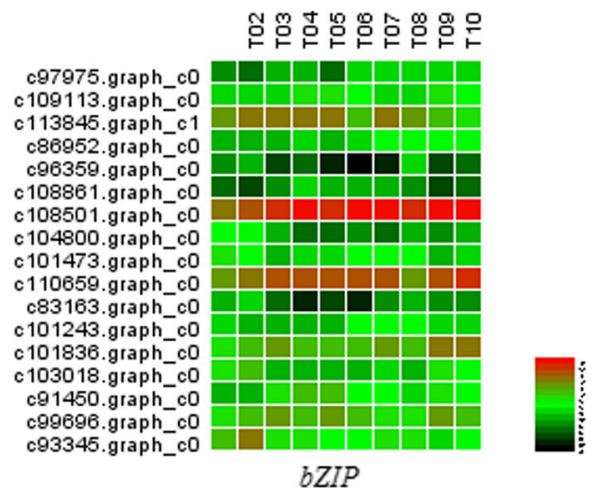

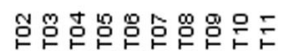

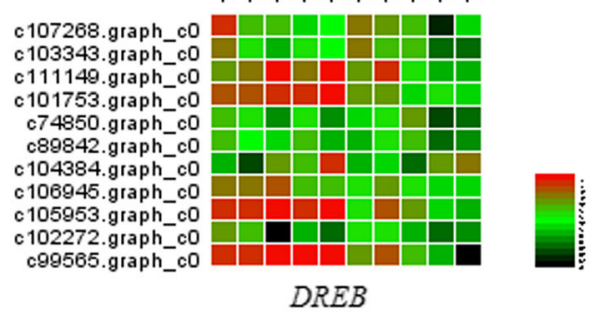

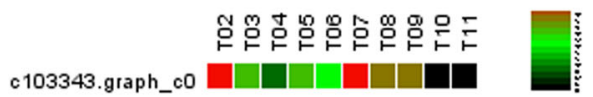

$C B F$

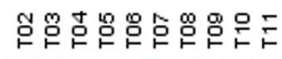

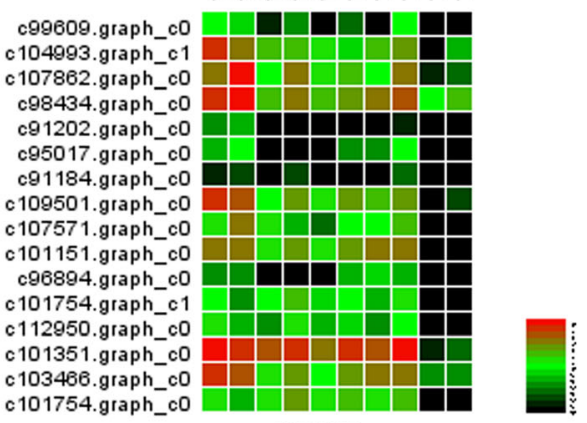

WRKY

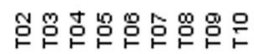

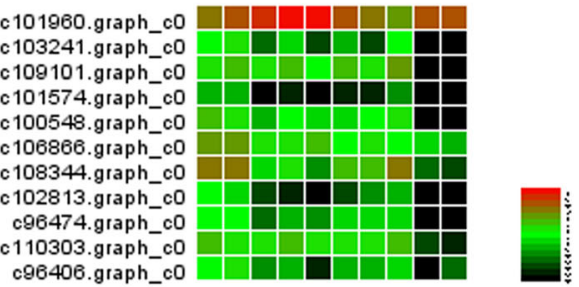

MYB

Fig. 10 The heat map of related transcription factors

inner membrane system of cells that play an important role in material transport. The increase in the layers of Golgi vesicles and the complexity of the structure showed that intracellular vesicle trafficking plays a role in the regulation and execution of bulb dormancy release [41]. As an important storage form of carbohydrates in lily bulbs, starch is often present in the form of starch granules. The number of starch granules in bulb cells decreased gradually during low temperature storage, which indicated that starch is continuously degraded in response to dormancy release.

\section{Changes in gene expression levels during low temperature storage \\ The key regulatory pathways were in response to dormancy release}

A shift in the relationship between sugar, starch and soluble sugar was found in studies of lily cryopreservation. The soluble sugar content was used as a judge to break dormancy and acts as a signaling molecule to regulate the growth of buds [42, 43]. Therefore, such starch-sugar interconversions might be mediated, in part, by the coaction of AMY, BMY and sucrose synthase (SuSy). The up-regulated DEGs related to AMY and BMY indicated that starch is degraded to provide metabolic energy for dormancy release and subsequent vegetative growth. In many studies, the increase in soluble sugar content is highly correlated with the enhancement in cold tolerance [44]. The DEGs related to SuSy were up-regulated in response to cold, water deficit, hypoxia and salt stress. Furthermore, SuSy simulated hypoxic conditions and cues in mitochondria together with pyruvate decarboxylase and alcohol dehydrogenase, to participate in the regulation of oxidative stress and dormancy release in plants [45]. Therefore, sucrose acts as an induction signal and is involved in not only dormancy release but also the formation of the protection mechanism under stress conditions.

The transition from dormancy to bud growth needs to consume energy. In grapes, Ophir et al. [46] suggested that $\mathrm{HC}$ - and heat shock (HS)-induced dormancy release involves the TCA cycle and ATP synthesis. Gai et al. [6] found several key enzymes that participated in the TCA cycle were up-regulated during artificial chilling induced dormancy release of Paeonia ostii. c11080 5.graph_c0 and c89446.graph_c0, encoding the main enzymes, 2-oxoglutarate dehydrogenase and isocitrate dehydrogenase, respectively, were up-regulated in S1 vs S4, which indicated that the TCA cycle is induced. Our finding was consistent with the enzyme activities during chilling induced dormancy release in Curcuma longa and Dioscorea esculenta [47]. Dormancy release 
is a biological process of energy consumption. The energy released by aerobic metabolism meets the need for dormancy release, and metabolism produces a variety of intermediates that provide compound carbon framework for the growth of cells after dormancy release [6, 47]. The PPP was also activated at the later stage of low temperature storage. Previous studies have found that PPP activation is associated with dormancy release both in perennial buds and seeds [24]. In addition, the defence response produced by plants under low temperature conditions can also induce the PPP, which provide antioxidant protection against ROS by producing NADPH [47]. The activation of the PPP is not only a signal of dormancy release, but also a protective mechanism under plant stress conditions. However, the down-regulated DEGs that were involved in glycolysis/gluconeogenesis accounted for more proportion. Glycolysis / gluconeogenesis may be inhibited. These results were not consistent with the response of Paeonia ostii to artificial cold treatment [6], but agreed with the studies of grapes and other dormant plants $[46,48]$.

Crosstalk among plant hormones coordinates the regulation of flowering and fruiting, maturation and senescence, as well as the dormancy and germination processes. The balance between ABA and GA determines plant dormancy [20]. The ABA/GA balance is disturbed when the plant undergoes environmental changes; low temperature conditions were associated with low ABA biosynthesis, which has an antagonistic effect to the GA response in numerous physiological processes [49] .Our data showed that the DEGs related to ABA synthesis, including NCED and CCD, were enriched in S1 vs S4 and were down-regulated. Meanwhile, GA2ox1 and GA3ox4, which are involved in GA degradation, were also downregulated. GA may dominate to promote bulb dormancy release under low temperature conditions. ETH actively regulates dormancy release by reducing $\mathrm{ABA}$ synthesis, enhancing $\mathrm{ABA}$ degradation, or changing $\mathrm{ABA}$ activity [50]. Ophir et al. [46] found that the DEGs related to ETH biosynthesis and signal transduction were up-regulated during grape bud dormancy release. In our study, c97695.graph_c0 encoding AC0, was up-regulated to increase the ETH synthesis level, which agreed with previous studies. It was reported that SA promoted dormancy release by measuring $\mathrm{H}_{2} \mathrm{O}_{2}$ production in grapes [51]. However, Nugroho et al. [52] noted that the differential expression associated with SA genes was not caused by plant dormancy but be more closely related to anthocyanins in the phenylpropanoid pathway and changes in flavonoid biosynthesis. In Arabidopsis mutants, germination rates correlate strongly with elevated JA levels [53]. Our data showed that the levels of JA and SA were higher in the early stage of low temperature storage. Increases in JA and SA concentrations were associated with plant responses to stress and the development of the vegetative axis [54].There is no clear consensus for the influence of JA / SA on dormancy regulation.

\section{The genes were differentially expressed to adapt to low temperature conditions}

Dormancy release induction by natural or artificial stress signals (such as low temperature) was associated with the antioxidation reaction [55]. ROS interacted with ABA and GA transduction pathway and were likely to control numerous transcription factors and properties of specific proteins through their carbonylation [56]. CAT is responsible for the removal of excess ROS during stress. It was reported that CAT inhibition accelerated dormancy release and sprouting in potatoes [57]. In our study, c104193.graph_c1, a gene encoding CAT, was downregulated in S1 vs S4. CAT inhibition could result in an increase in the level of endogenous $\mathrm{H}_{2} \mathrm{O}_{2}$ in bulb tissues, which might activate PPP and thus lead to dormancy release and initiation of sprouting [58]. In addition, several main enzymes that were involved in the antioxidant reaction, including ADH, StSy and GST, were up-regulated at the later stage of low temperature storage, which was similar to antioxidant enzyme changes of Paeonia ostii during artificial chilling induced dormancy release [6]. These results indicate that bulb dormancy release induced by cold acclimation is associated with the removal of free radicals through activated peroxide-scavenging systems.

The plant responds to internal or external environmental signals through epigenetic modifications, including DNA methylation [59]. Previous studies have reported that the low temperature associated changes in DNA methylation are evident [60]. Kumar et al. [27] found that cold acclimation during dormancy in the apple was likely to affect the epigenetic regulation through DNA methylation. Our data showed that 68 genes were involved in DNA methylation; therefore, bulbs could modulate growth and development to adapt under cold stress with epigenetic mechanisms. The DEGs encoding DNA (cytosine-5)-methyltransferase were down-regulated in S1 vs S4. This finding was consistent with the analysis of Bai et al. [13]. With prolonged cold storage, the level of methylation decreased, which suggested that epigenetic regulation participates in bulb dormancy release.

\section{The regulatory mechanism of dormancy release during low temperature storage \\ The correlation between changes in bulb cell ultrastructures and gene expression levels}

From ultrastructural observation, we found that the shape of mitochondria changed from a long stick to a sphere during low temperature storage. Mitochondria may divide to increase the abundance in number. Mitochondria are the main sites for aerobic respiration, which provides energy to meet the needs of dormancy release. Meanwhile, 


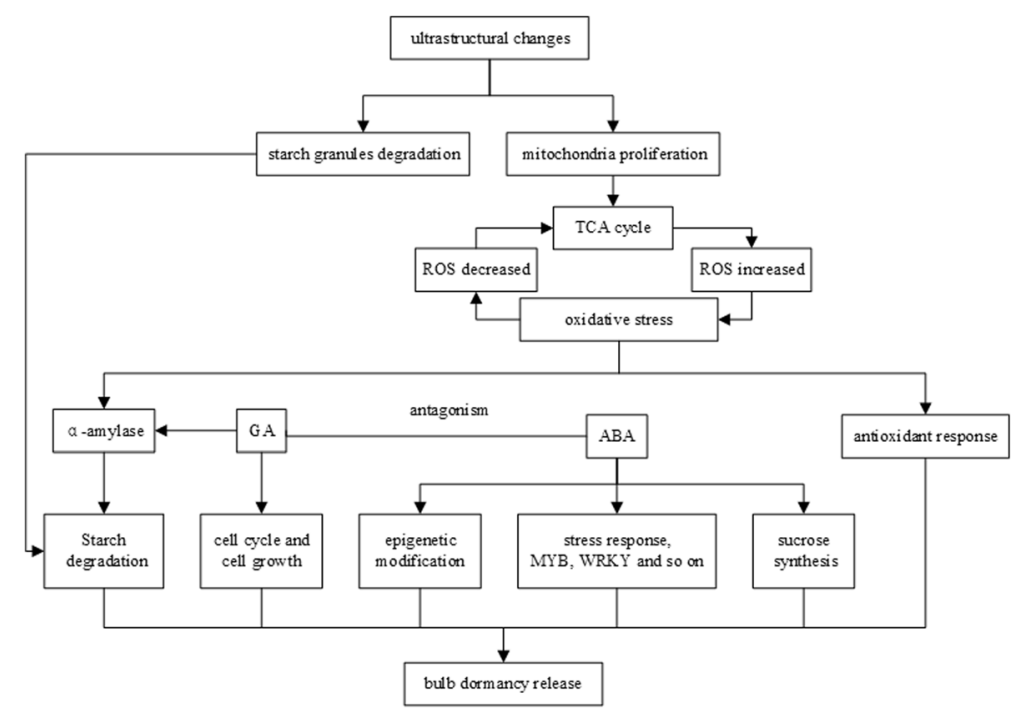

Fig. 11 The regulatory mechanism of dormancy release during low temperature storage of Lilium pumilum bulbs

mitochondria are also important sources of ROS production [61]. With increased levels of ROS, many antioxidant enzymes such as CAT, ADH, StSy and GST, are activated. Therefore, peroxide-scavenging systems occurs [6]. In turn, the transcription and metabolite levels in the TCA cycle are reduced with the increased levels of ROS [46], thereby maintaining proper ROS concentrations and promoting dormancy release (Fig. 11).

In plants, sugar (or carbohydrate compounds) and phenolic compounds act as part of the redox system, removing ROS and contributing to stress tolerance [62]. Transient oxidative stress and respiratory stress may activates AMY, accelerating the hydrolysis of starch [63]. In addition, the activation of GA biosynthesis and signalling pathway by artificial chilling could also induce AMY gene expression [64]. Ultrastructural observation revealed that parenchyma cells were completely filled with starch granules at the initial time of low temperature storage, which significantly decreased in number after $90 \mathrm{~d}$ of cold storage. Correspondingly, the DEGs related to AMY were activated. The morphological changes of cells and the differential expression of related genes were consistent, which also suggested that starch hydrolysis coupled with the antioxidant reaction and GA signalling pathway was involved in bulb dormancy release (Fig. 11).

\section{The regulatory network constituted by DEGs}

The antagonism of ABA and GA determines plants dormancy [20]. It was reported that the expression of SuSy gene can respond to changes in endogenous $A B A$ at transcription and post - transcriptional levels [65]. Sugar interacted with ABA signalling pathways in seeds [66]. Moreover, GA acted as a positive regulator of the cell cycle and cell growth, transforming the mitotic cycle to endocycle during cell differentiation, thereby affecting cell elongation [67]. It can be concluded that the biological processes including glucose metabolism, plant hormone signalling pathway, cell division and growth, are coupled with each other during bulb dormancy release (Fig. 11).

$\mathrm{ABA}$ is a broad-spectrum phytohormone involved not only in regulating growth and development but also in coordinating various stress signal transduction pathways when plants are under abiotic stress conditions [68]. The ABA signal transduction pathways from stress signal perception to gene expression involve different transcription factors, including DREB, MYB, WRKY and $b Z I P$, during bulb cold storage. It has been reported that epigenetic processes are an integral part of ABAregulated processes [69]. Bulb cells need to adapt to cold stress during low temperature storage, epigenetic modification and the differential expression of various transcription factors are in response to ABA signalling, therefore regulating dormancy release (Fig. 11).

\section{Conclusions}

Morphological changes reflect the process of dormancy release in bulbs; transcriptome sequencing provides us with comprehensive sequences and DGE analysis data. This study enriches the genome information of L. pumilum and lays the foundation for exploring the mechanism of bulb dormancy.

\section{Methods}

Plant materials

The test materials were three-year-old bulbs of L. pumilum, with a circumference of $4-6 \mathrm{~cm}$ that were planted in 
the nursery of Northeast Forestry University and managed conventionally. The disease- and pest-free bulbs of $L$. pumilum were harvested in mid-October 2014. After $30 \mathrm{~min}$ of sterilization with $50 \%$ carbendazim WP 800 times aqueous solution, the samples were thoroughly washed and dried. Wet steam-sterilized perlite was used as the storage matrix, and the bulbs were placed in a refrigerator at $4^{\circ} \mathrm{C}$ for cold storage. Samples stored for 0, 30, 60 , and 90 days were labelled as S1, S2, S3 and S4, respectively; they were used in the study of ultrastructure and transcriptome sequencing.

\section{Ultrastructure of bulb cells}

Shoot apical meristem samples of bulbs were cut into $0.5-1 \mathrm{~mm}^{3}$ pieces and fixed in $2.5 \%$ glutaraldehyde fixative (vacuumed to submerge) at $4{ }^{\circ} \mathrm{C}$ for at least $24 \mathrm{~h}$. The tissue materials were washed with $0.1 \mathrm{~mol} \cdot \mathrm{L}-1$ phosphate buffer for $2 \mathrm{~h}$, and then dehydrated with 30\%, 50\%, 70\%, $80 \%, 90 \%$ and $100 \%$ ethanol; the dehydrated samples were infiltrated and embedded with Epon812. The samples were sliced with an ULTRACUTE ultrathin microtome and stained with uranyl acetate and lead citrate. The tissue sections were examined with an H-7650 transmission electron microscope. Three fields were selected and five cells in each field were examined for ultrastructural changes in the bulb cells and imaged.

\section{Total RNA isolation, library construction and transcriptome sequencing}

Total RNA was extracted using the EASYspin Plus Plant RNA Rapid Extraction Kit (Cat. RN38). The quality of RNA was measured with a micro-spectrophotometer Nanodrop 2000 and agarose gel electrophoresis. RNA purity was measured by a NanoPhotometer ${ }^{\bullet}$ spectrophotometer (IMPLEN, CA, USA). RNA concentration was measured with the Qubit ${ }^{\oplus}$ RNA Assay Kit in Qubit $^{\bullet} 2.0$ Flurometer (Life Technologies, CA, USA). The concentration of RNA was $400 \mathrm{ng} \cdot \mu \mathrm{L}^{-1}$, and it had an A260:280 ratio of 1.8 to 2.0. The gel electrophoresis strips were clear with no tailing, and the brightness ratio of $28 \mathrm{~S}$ to $18 \mathrm{~S}$ was $2: 1$. The RNA quality met sequencing requirements. The cDNA library was sequenced using the Illumina HiSeq 2500 high throughput sequencing platform, and a large number of high-quality reads were obtained. Most of the base quality scores met or exceeded Q30. Sequences were assembled withTrinity [70] to construct a sequencing library.

\section{Gene functional annotation and expression analysis}

Unigene annotation information was obtained by comparing the unigene sequences to $\mathrm{nr}$ [71], Swiss-Prot [72], GO [73], COG [74], and KEGG [75] databases with BLAST [76] software (version 2.2.26). Eventually, 29,811 annotated unigenes were obtained.
The reads from each sequencing sample were compared to the unigene database with Bowtie [77]. The expression levels were estimated by RSEM [78] based on the alignment results. The expression levels of genes were based on FPKM [79], and the FPKM formula is as follows:

$$
F P K M=\frac{c D N A \text { Fragments }}{\text { Mapped Fragments (Millions }) \times \text { Transcript Length }(k b)}
$$

\section{Differential expression analysis, DEGs functional} annotation and enrichment analysis

The Pearson correlation coefficient was used as an indicator of correlation between biological replicates [80], and the samples were highly correlated. The differences of expression between two groups were analysed by DESeq [81] and FDR (False Discovery Rate) $<0.01$ and a fold change (the ratio of expression between two groups) $\geq 2$ were used as the criteria to screen DEGs. Hierarchical cluster analysis was used to show the expression patterns of genes with the same or similar expression behaviour, and the DEGs were compared with GO, COG and KEGG databases for functional enrichment analysis.

\section{Validation by qRT-PCR}

Fourteen DEGs were randomly selected for qRT-PCR. Primer information was shown in Additional file 1. Reactions were performed with Roche LightCycler 96: Preincubation $\left(95^{\circ} \mathrm{C}\right.$ for $\left.30 \mathrm{~s}\right)$; three step amplification $\left(95^{\circ}\right.$ $\mathrm{C}$ for $5 \mathrm{~s} ; 60{ }^{\circ} \mathrm{C}$ for $15 \mathrm{~s} ; 72{ }^{\circ} \mathrm{C}$ for $30 \mathrm{~s} ; 40$ cycles); melting $\left(95{ }^{\circ} \mathrm{C}\right.$ for $10 \mathrm{~s} ; 65^{\circ} \mathrm{C}$ for $60 \mathrm{~s} ; 97^{\circ} \mathrm{C}$ for $1 \mathrm{~s}$ ); cooling $\left(37{ }^{\circ} \mathrm{C}\right.$ for $30 \mathrm{~s}$ ). All experiments were done with three biological replicates. Relative expression was calculated according to the $2^{-\Delta \Delta C t}$ algorithm, and was based on expression of the actin gene (JX826390).

\section{Additional files}

Additional file 1: The unigene length distribution. (XLSX 9 kb) Additional file 2: Statistics for de novo assembly of transcriptome. (XLSX 9 kb)

Additional file 3: GO enrichment analysis of DEGs. (XLS 33 kb) Additional file 4: DEGs involved in KEGG pathways. (XLS $72 \mathrm{~kb}$ ) Additional file 5: The key regulatory pathways involved in dormancy release. (XLSX $15 \mathrm{~kb}$ )

Additional file 6: DEGs involved in cell activity during dormancy release. (XLSX $13 \mathrm{~kb})$

Additional file 7: qRT-PCR primer information. (XLS 24 kb)

Abbreviations

ABA: Abscisic acid; DEGs: Differentially expressed genes; DGE: Digital Gene Expression Tag Profiling; ETH: Ethylene; GA: gibberellin; ROS: Reactive oxygen species 


\section{Acknowledgements}

We thank Prof. Yunwei Zhou and Miao He for their help and support, we would like to acknowledge Zhongping Tian and Yu Liu for guidance and sincerely thank the editors and reviewers for their patient work.

\section{Funding}

This study was financially supported by National Natural Science Foundation of China (31470698). The funding body had no role in the design and collection, analysis and interpretation of the data.

\section{Availability of data and materials}

All the data supporting our findings are contained within the manuscript.

\section{Authors' contributions}

WW and XXS designed the research and wrote the manuscript. ZPT and YL provided guidance on the experiment. YWZ and MH reviewed and revised the manuscript. All authors read and approved the final manuscript.

\section{Ethics approval and consent to participate}

Not applicable.

\section{Consent for publication}

Not applicable.

\section{Competing interests}

The authors declare that they have no competing interests.

\section{Publisher's Note}

Springer Nature remains neutral with regard to jurisdictional claims in published maps and institutional affiliations.

Received: 26 November 2017 Accepted: 6 February 2018 Published online: 14 March 2018

\section{References}

1. Bachem C, vdHR LJ, Oomen R, Casarini E, Jacobsen E, Visser R. Functional genomic analysis of potato tuber life-cycle. Potato Res. 2000;43(4):297-312.

2. Verma V, Ravindran P, Kumar P P. Plant hormone-mediated regulation of stress responses. Bmc Plant Biology. 2016;16(1):86.

3. Alonso-Ramírez A, Rodríguez D, Reyes $D$, Jiménez JA, Nicolás G, LópezCliment M, Gómez-Cadenas A, Nicolás C. Evidence for a role of gibberellins in salicylic acid-modulated early plant responses to abiotic stress in Arabidopsis seeds. Plant Physiol. 2009;150(3):1335-44.

4. KeÇpczyński J, KeÇpczyńska E. Ethylene in seed dormancy and germination. Physiol Plant. 1997;101(4):720-6.

5. Ghassemian M, Nambara E, Cutler S, Kawaide H, Kamiya Y, McCourt P. Regulation of abscisic acid signaling by the ethylene response pathway in Arabidopsis. Plant Cell. 2000;12(7):1117-26.

6. Gai S, Zhang Y, Liu C, Zhang Y, Zheng G. Transcript profiling of Paoenia ostii during artificial chilling induced dormancy release identifies activation of GA pathway and carbohydrate metabolism. PLoS One. 2013;8(2):e55297.

7. Oracz K, El-Maarouf-Bouteau H, Kranner I, Bogatek R, Corbineau F, Bailly C. The mechanisms involved in seed dormancy alleviation by hydrogen cyanide unravel the role of reactive oxygen species as key factors of cellular signaling during germination. Plant Physiol. 2009;150(1):494-505.

8. Kwak JM, Nguyen V, Schroeder JI. The role of reactive oxygen species in hormonal responses. Plant Physiol. 2006:141(2):323-9.

9. Rushton PJ, Somssich IE, Ringler P, Shen QJ. WRKY transcription factors. Trends Plant Sci. 2010;15(5):247-58

10. Wisniewski M, Norelli J, Artlip T. Overexpression of a peach CBF gene in apple: a model for understanding the integration of growth, dormancy, and cold hardiness in woody plants. Front Plant Sci. 2015;6:85.

11. Cadman CS, Toorop PE, Hilhorst HW, Finch-Savage WE. Gene expression profiles of Arabidopsis cvi seeds during dormancy cycling indicate a common underlying dormancy control mechanism. Plant J. 2006;46(5):805-22.

12. Habu T, Yamane H, Igarashi K, Hamada K, Yano K, Tao R. 454pyrosequencing of the transcriptome in leaf and flower buds of Japanese apricot (Prunus mume Sieb. Et Zucc.) at different dormant stages. J Jpn Soc Horticultural Sci. 2012;81(3):239-50.
13. Bai S, Saito T, Sakamoto D, Ito A, Fujii H, Moriguchi T. Transcriptome analysis of Japanese pear (Pyrus pyrifolia Nakai) flower buds transitioning through endodormancy. Plant Cell Physiol. 2013;54(7):1132-51.

14. Liu G, Li W, Zheng P, Tong X, Chen L, Liu D, Hussain S, Teng Y. Transcriptomic analysis of 'Suli' pear (Pyrus pyrifolia white pear group) buds during the dormancy by RNA-Seq. BMC Genomics. 2012;13(1):700.

15. Yamauchi Y, Ogawa M, Kuwahara A, Hanada A, Kamiya Y, Yamaguchi S. Activation of gibberellin biosynthesis and response pathways by low temperature during imbibition of Arabidopsis thaliana seeds. Plant Cell. 2004;16(2):367-78.

16. Langens-Gerrits MMMW, Croes AF, de Klerk G-J. Effect of low temperature on dormancy breaking and growth after planting in lily bulblets regenerated in vitro. Plant Growth Regul. 2003;40(3):267-75.

17. Pitschmann A, Purevsuren S, Obmann A, Natsagdorj D, Gunbilig D, Narantuya S, Kletter C, Glasl S. Traditional mongolian medicine: history and status quo. Phytochem Rev. 2013;12(4):943-59.

18. Kaplan F, Sung DY, Guy CL. Roles of $\beta$-amylase and starch breakdown during temperatures stress. Physiol Plant. 2006;126(1):120-8.

19. Xu R-Y, Niimi Y, Han D-S: Changes in endogenous abscisic acid and soluble sugars levels during dormancy-release in bulbs of Lilium rubellum. Sci Hortic 2006; 111(1):68-72.

20. Liu F, Zhang H, Wu G, Sun J, Hao L, Ge X, Yu J, Wang W. Sequence variation and expression analysis of seed dormancy- and germination-associated ABA- and GA-related genes in rice cultivars. Front Plant Sci. 2011;2:17.

21. Howe GT, Horvath DP, Dharmawardhana P, Priest HD, Mockler TC, Strauss $\mathrm{SH}$. Extensive transcriptome changes during natural onset and release of vegetative bud dormancy in Populus. Front Plant Sci. 2015;6:989.

22. Leida C, Terol J, Marti G, Agusti M, Llacer G, Badenes ML, Rios G. Identification of genes associated with bud dormancy release in Prunus persica by suppression subtractive hybridization. Tree Physiol. 2010;30(5): 655-66.

23. Zhu Y, Li Y, Xin D, Chen W, Shao X, Wang Y, Guo W. RNA-Seq-based transcriptome analysis of dormant flower buds of Chinese cherry (Prunus pseudocerasus). Gene. 2015;555(2):362-76.

24. Halaly T, Pang X, Batikoff T, Crane O, Keren A, Venkateswari J, Ogrodovitch A, Sadka A, Lavee S, Or E. Similar mechanisms might be triggered by alternative external stimuli that induce dormancy release in grape buds. Planta. 2008;228(1):79-88.

25. Zhang L, Wang Y, Zhang X, Zhang M, Han D, Qiu C, Han Z. Dynamics of phytohormone and DNA methylation patterns changes during dormancy induction in strawberry (Fragaria x ananassa Duch.). Plant Cell Rep. 2012; 31(1):155-65.

26. Law RD, Suttle JC. Transient decreases in methylation at 5'-cCGG-3' sequences in potato (Solanum tuberosum L.) meristem DNA during progression of tubers through dormancy precede the resumption of sprout growth. Plant Mol Biol. 2003;51(3):437-47.

27. Kumar G, Rattan UK, Singh AK. Chilling-mediated DNA methylation changes during dormancy and its release reveal the importance of epigenetic regulation during winter dormancy in apple (Malus $\mathrm{x}$ domestica Borkh.). PLoS One. 2016;11(2):e0149934.

28. Devitt ML, Stafstrom JP. Cell cycle regulation during growth-dormancy cycles in pea axillary buds. Plant Mol Biol. 1995;29(2):255-65.

29. Berckmans B, De Veylder L. Transcriptional control of the cell cycle. Curr Opin Plant Biol. 2009;12(5):599-605.

30. Agarwal M, Hao Y, Kapoor A, Dong CH, Fujii H, Zheng X, Zhu JK. A R2R3 type MYB transcription factor is involved in the cold regulation of $C B F$ genes and in acquired freezing tolerance. J Biol Chem. 2006;281(49):37636-45.

31. Abe H, Urao T, Ito T, Seki M, Shinozaki K, Yamaguchi-Shinozaki K. Arabidopsis AtMYC2 (bHLH) and AtMYB2 (MYB) function as transcriptional activators in abscisic acid signaling. Plant Cell. 2003;15(1):63.

32. Rushton DL, Tripathi P, Rabara RC, Lin J, Ringler P, Boken AK, Langum TJ, Smidt L, Boomsma DD, Emme NJ, et al. WRKY transcription factors: key components in abscisic acid signalling. Plant Biotechnol J. 2012;10(1):2-11.

33. Griffith M, Yaish MW. Antifreeze proteins in overwintering plants: a tale of two activities. Trends Plant Sci. 2004;9(8):399-405.

34. Amir Hossain M, Lee $\mathrm{Y}$, Cho Jl, Ahn CH, Lee SK, Jeon JS, Kang H, Lee CH, An $G$, Park PB. The bZIP transcription factor OSABF1 is an ABA responsive element binding factor that enhances abiotic stress signaling in rice. Plant Mol Biol. 2010;72(4-5):557-66.

35. Lata C, Prasad M. Role of DREBs in regulation of abiotic stress responses in plants. J Exp Bot. 2011;62(14):4731-48. 
36. Jian L-C, Li PH, Sun L-H, Chen THH. Alterations in ultrastructure and subcellular localization of $\mathrm{Ca} 2+$ in poplar apical bud cells during the induction of dormancy. J Exp Bot. 1997;48(6):1195-207.

37. Chien C-T, Kuo-Huang L-L, Lin T-P. Changes in ultrastructure and abscisic acid level, and response to applied gibberellins in Taxus mairei seeds treated with warm and cold stratification. Ann Bot. 1998:81(1):41-7.

38. Kratsch HA, Wise RR. The ultrastructure of chilling stress. Plant Cell Environ. 2000;23(4):337-50.

39. Rosen ED, Spiegelman BM. PPAR gamma: a nuclear regulator of metabolism, differentiation, and cell growth [review]. J Biol Chem. 2001;276(41):37731-4.

40. Huang X, Zhu W, Dai S, Gai S, Zheng GC. The involvement of mitochondrial phosphate transporter in accelerating bud dormancy release during chilling treatment of tree peony (Paeonia suffruticosa). Planta. 2008;228(4):545-52.

41. Keilin T, Pang X, Venkateswari J, Halaly T, Crane O, Keren A, Ogrodovitch A, Ophir R, Volpin H, Galbraith D, et al. Digital expression profiling of a grapebud EST collection leads to new insight into molecular events during grape-bud dormancy release. Plant Sci. 2007;173(4):446-57.

42. Chao WS, Serpe MD. Changes in the expression of carbohydrate metabolism genes during three phases of bud dormancy in leafy spurge. Plant Mol Biol. 2010;73(1-2):227-39.

43. Liu X, Wang Q, Gu J, Lu Y. Vernalization of oriental hybrid lily 'Sorbonne': changes in physiology metabolic activity and molecular mechanism. Mol Biol Rep. 2014;41(10):6619-34

44. Parker J. Relationships among cold hardiness, water-soluble protein, anthocyanins, \&free sugars in Hedera helix L. Plant Physiol. 1962;37(6):809.

45. Kolman MA, Torres LL, Martin ML, Salerno GL. Sucrose synthase in unicellular cyanobacteria and its relationship with salt and hypoxic stress. Planta. 2012;235(5):955-64.

46. Ophir R, Pang X, Halaly T, Venkateswari J, Lavee S, Galbraith D, Or E. Geneexpression profiling of grape bud response to two alternative dormancyrelease stimuli expose possible links between impaired mitochondrial activity, hypoxia, ethylene-ABA interplay and cell enlargement. Plant $\mathrm{Mo}$ Biol. 2009;71(4-5):403-23.

47. Panneerselvam R, Abdul Jaleel C, Somasundaram R, Sridharan R, Gomathinayagam M. Carbohydrate metabolism in Dioscorea esculenta (Lour. ) Burk. Tubers and Curcuma longa L. rhizomes during two phases of dormancy. Colloids Surf B: Biointerfaces. 2007;59(1):59-66.

48. Wang SY, Jiao HJ, Faust M. Changes in metabolic enzyme activities during thidiazuron-induced lateral budbreak of apple. HortSci. 1991;26(2):171-3.

49. Shu K, Chen Q, Wu Y, Liu R, Zhang H, Wang P, Li Y, Wang S, Tang S, Liu C. ABI4 mediates antagonistic effects of abscisic acid and gibberellins at transcript and protein levels. Plant Jo Cell Mol Biol. 2016;85(3):348.

50. Huo H, Bradford $\mathrm{KJ}$ : Molecular and hormonal regulation of thermoinhibition of seed germination. 2015

51. Or E, Vilozny I, Eyal Y, Ogrodovitch A. The transduction of the signal for grape bud dormancy breaking induced by hydrogen cyanamide may involve the SNF-like protein kinase GDBRPK. Plant Mol Biol. 2000;43(4):483-94.

52. Alon M, Malka O, Eakteiman G, Elbaz M, Ben M, Zvi M, Vainstein A, Morin S. Activation of the phenylpropanoid pathway in Nicotiana tabacum improves the performance of the whitefly Bemisia tabaci via reduced jasmonate signaling. PLoS One. 2013:8(10):e76619.

53. Andrade A, Riera N, Lindstrom L, Alemano S, Alvarez D, Abdala G, Vigliocco A. Pericarp anatomy and hormone profiles of cypselas in dormant and non-dormant inbred sunflower lines. Plant Biol (Stuttgart, Germany). 2015:17(2):351-60.

54. Ribeiro LM, Garcia QS, Müller M, Munnébosch S. Tissue-specific hormonal profiling during dormancy release in macaw palm seeds. Physiol Plant. 2015;153(4):627

55. Walton EF, Wu R-M, Richardson AC, Davy M, Hellens RP, Thodey K, Janssen $\mathrm{BJ}$, Gleave AP, Rae GM, Wood M. A rapid transcriptional activation is induced by the dormancy-breaking chemical hydrogen cyanamide in kiwifruit (Actinidia deliciosa) buds. J Exp Bot. 2009:60(13):3835-48.

56. El-Maarouf-Bouteau H, Bailly C. Oxidative signaling in seed germination and dormancy. Plant Signal Behav. 2008;3(3):175-82.

57. Bajji M, M'Hamdi M, Gastiny F, Rojasbeltran JA. Catalase inhibition accelerates dormancy release and sprouting in potato (Solanum tuberosum L.) tubers. Biotechnologie Agronomie Société Et Environnement. 2007;11(2):121-31.

58. Fontaine O, Huault C, Pavis N, Billard JP. Dormancy breakage of Hordeum vulgare seeds: effects of hydrogen peroxide and scarification on glutathione level and glutathione reductase activity. Plant Physiol Biochem. 1994;32(5):677-83.
59. Grimanelli D, Roudier F. Epigenetics and development in plants: green light to convergent innovations. Curr Top Dev Biol. 2013;104:189-222.

60. Guzywrobelska J, Filek M, Kaliciak A, Szarejko I, Macháčková I, Krekule J, Barciszewska M. Vernalization and photoperiod-related changes in the DNA methylation state in winter and spring rapeseed. Acta Physiol Plant. 2013; 35(3):817-27.

61. Murphy Michael P. How mitochondria produce reactive oxygen species. Biochem J. 2009;417(1):1-13

62. Bolouri-Moghaddam MR, Le Roy K, Xiang L, Rolland F, Van den Ende W. Sugar signalling and antioxidant network connections in plant cells. FEBS J. 2010;277(9):2022-37.

63. Rubio S, Donoso A, Pérez FJ. The dormancy-breaking stimuli "chilling, hypoxia and cyanamide exposure" up-regulate the expression of a-amylase genes in grapevine buds. J Plant Physiol. 2014;171(6):373-81.

64. Olszewski N, Gubler F. Gibberellin signaling: biosynthesis, catabolism, and response pathways. Plant Cell. 2002;14(90001):S61.

65. Koch K. Sucrose metabolism: regulatory mechanisms and pivotal roles in sugar sensing and plant development. Curr Opin Plant Biol. 2004;7(3):235-46.

66. Finkelstein RR, Gampala SSL, Rock CD. Abscisic acid signaling in seeds and seedlings. Plant Cell. 2002;14(suppl 1):S15-45.

67. Gendreau E, Orbovic V, Hofte H, Traas J. Gibberellin and ethylene control endoreduplication levels in the Arabidopsis thaliana hypocotyl. Planta. 1999; 209(4):513-6.

68. Agarwal PK, Jha B. Transcription factors in plants and ABA dependent and independent abiotic stress signaling. Biol Plant. 2010;54(2):201-12.

69. Chinnusamy V, Gong Z, Zhu JK. Abscisic acid-mediated epigenetic processes in plant development and stress responses. Chinese J Plant Ecol. 2008:50(10):1187-95.

70. Grabherr MG, Haas BJ, Yassour M, Levin JZ, Thompson DA, Amit I, Adiconis X, Fan L, Raychowdhury R, Zeng Q, et al. Full-length transcriptome assembly from RNA-Seq data without a reference genome. Nat Biotechnol. 2011;29(7): 644-52.

71. Li W, Jaroszewski L, Godzik A. Tolerating some redundancy significantly speeds up clustering of large protein databases. Bioinformatics (Oxford, England). 2002;18(1):77-82

72. Apweiler R, Bairoch A, Wu CH, Barker WC, Boeckmann B, Ferro S, Gasteige E, Huang H, Lopez R, Magrane M, et al. UniProt: the universal protein knowledgebase. Nucleic Acids Res. 2004;32(Database issue):D115-9.

73. Ashburner M, Ball CA, Blake JA, Botstein D, Butler H, Cherry JM, Davis AP, Dolinski K, Dwight SS, Eppig JT, et al. Gene ontology: tool for the unification of biology. The gene ontology consortium. Nat Genet. 2000;25(1):25-9.

74. Tatusov RL, Galperin MY, Natale DA, Koonin EV. The COG database: a tool for genome-scale analysis of protein functions and evolution. Nucleic Acids Res. 2000:28(1):33-6.

75. Kanehisa M, Goto S, Kawashima S, Okuno Y, Hattori M. The KEGG resource for deciphering the genome. Nucleic Acids Res. 2004;32(Database issue): D277-80.

76. Altschul SF, Madden TL, Schaffer AA, Zhang J, Zhang Z, Miller W, Lipman DJ, Gapped BLAST. PSI-BLAST: a new generation of protein database search programs. Nucleic Acids Res. 1997;25(17):3389-402.

77. Langmead B, Trapnell C, Pop M, Salzberg SL. Ultrafast and memory-efficient alignment of short DNA sequences to the human genome. Genome Biol. 2009;10(3):R25.

78. CND BL. RSEM. Accurate transcript quantification from RNA Seq data with or without a reference genome. BMC Bioinformatics. 2011:12:323.

79. Trapnell C, Williams BA, Pertea G, Mortazavi A, Kwan G, van Baren MJ, Salzberg SL, Wold BJ, Pachter L. Transcript assembly and quantification by RNA-Seq reveals unannotated transcripts and isoform switching during cell differentiation. Nat Biotechnol. 2010;28(5):511-5.

80. Schulze SK, Kanwar R, Golzenleuchter M, Therneau TM, Beutler AS. SERE: single-parameter quality control and sample comparison for RNA-Seq. BMC Genomics. 2012;13:524.

81. Anders S, Huber W. Differential expression analysis for sequence count data. Genome Biol. 2010;11(10):R106. 